\title{
Tripod-shaped Syndactyly in Apert Syndrome with FGFR2 p.P253R Mutation
}

\author{
Chandra Bhan Singh ${ }^{1} \quad$ Biswajit Mishra ${ }^{2} \quad$ Rashmi Patel $^{1} \quad$ Ashok Kumar $^{3} \quad$ Akhtar Ali $^{1}$
}

${ }^{1}$ Centre for Genetic Disorders, Institute of Science, Banaras Hindu University, Varanasi, Uttar Pradesh, India

2Department of Plastic Surgery, MKCG. Medical College and Hospital, Berhampur, Odisha, India

${ }^{3}$ Department of Pediatrics, SS Hospital, Institute of Medical

Sciences, Banaras Hindu University, Varanasi, Uttar Pradesh, India

\author{
Address for correspondence Akhtar Ali, MSc, PhD, Centre for \\ Genetic Disorders, Institute of Science, Banaras Hindu University, \\ Varanasi 221005, Uttar Pradesh, India \\ (e-mail: akhtar_genetics@yahoo.co.in, akhtar@bhu.ac.in).
}

Indian J Plast Surg 2021;54:370-372.

\begin{abstract}
Keywords

- Apert syndrome

- tripod-shaped syndactyly

- FGFR2

$-758 \mathrm{C}>\mathrm{G}$

- Pro253Arg

- India

Apert syndrome is a rare acrocephalosyndactyly (craniosynostosis) syndrome characterized by craniofacial dysmorphism and syndactyly of the hands and feet. It is caused by FGFR2 mutations and inherited in an autosomal dominant manner. This article describes a novel clinical variant of Apert syndrome having bilateral symmetrical tripod-shaped syndactyly in hands with milder craniofacial features in a sporadic case, along with a mutation in the fibroblast growth factor receptor 2 (FGFR2) gene. The patient had shown craniosynostosis, dysmorphic face, ocular hypertelorism, marked depression of the nasal bridge, long philtrum, and low set ears. Direct resequencing of the FGFR2 gene through Sanger's method identified a heterozygous missense mutation; FGFR2c.758C>C (FGFR2p.P253R) in the exon-7 of the gene.
\end{abstract}

\section{Introduction}

Congenital cranial deformity initially was described by S.W. Wheaton (1894) in two infants who also suffered from syndactyly of hands and feet. ${ }^{1}$ Later, Eugene Charles Apert reported a summary of nine similar cases and coined the term "de l'acrocephalosyndactylie" syndrome, which is commonly called acrocephalosyndactyly type I (ACS1) syndrome. ${ }^{2}$ It was later named as Apert syndrome (Online Mendelian Inheritance in Man database code: AS, OMIM 101200). Apert syndrome is a rare genetic condition inherited in an autosomal dominant pattern. Apert syndrome is characterized by craniosynostosis, dysmorphic face, and fusion of the fingers. Cleft palate and abnormalities in face, eye, dentition, mouthparts, central nervous system, and respiratory tract are also observed in Apert syndrome. ${ }^{3-10}$

Both males and females are affected equally in this syndrome. Its occurrence is approximately one out of 65,000 live

published online August 19, 2021
DOI https://doi.org/

$10.1055 / \mathrm{s}-0041-1733808$ ISSN 0970-0358 births and accounts for $\sim 4.5 \%$ of all the cases of craniosynostosis; the frequency is $\sim 1$ in 2,500 live births with genetic heterogeneity. Both familial and sporadic cases of Apert syndrome have been reported. ${ }^{4}$ The risk of having Apert syndrome significantly increases with paternal age due to the failure of selective advantage within the male spermatogonial cells.

Apert syndrome is caused by fibroblast growth factor receptor 2 (FGFR2) mutations in which two mutations p.S252W. (in 64\% cases) and P253R (in 33\% cases) are most common. Both p.S252W and p.P253R. mutations show complete penetrance with variable expressivity. ${ }^{4}$ More than 180 syndromes are known with the involvement of craniosynostosis, of which eight are associated with mutations in the FGFR2 gene. For example, Apert (OMIM 101200), Crouzon (OMIM 123500), Pfeiffer (OMIM 101600), Beare-Stevenson (OMIM 123790), and Jackson-Weiss (OMIM 123150) syndromes are caused by FGFR2 mutation. . $^{5-10}$ 


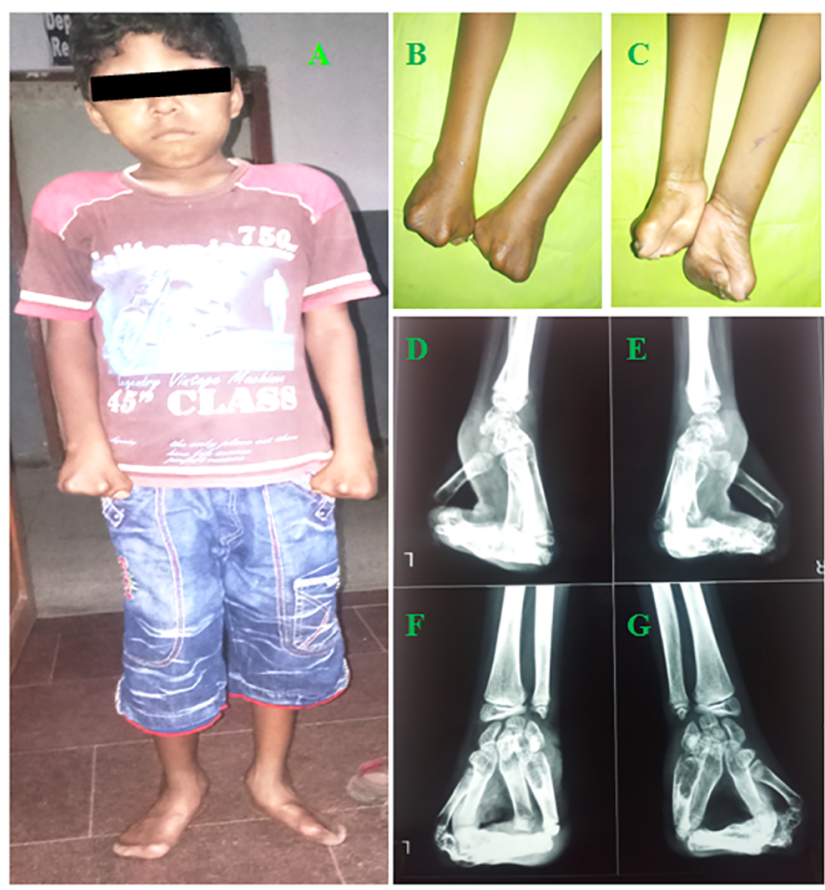

Fig. 1 Photographs showing external phenotypes of the patient and $\mathrm{X}$-ray radiographs of hands. (a) Facial abnormalities, and syndactyly in hands and feet. (b) Ventral and (c) dorsal view of both hands showing severe symmetrical and tripod-shaped syndactyly. Radiographs of left hand (d, f) and right hand $(\mathbf{e}, \mathbf{g})$.

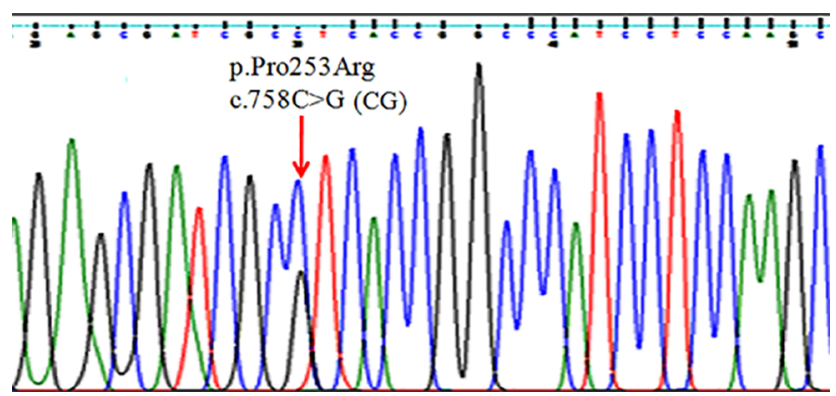

Fig. 2 DNA sequencing electropherogram of exon 7 of the FGFR2 gene showing missense heterozygous mutation p.P253R. (FGFR2C.758C>G).

\section{Materials and Methods}

Ethical approval was obtained from the institutional ethical committee. After written informed consent was obtained, peripheral blood $(4 \mathrm{~mL})$ was drawn in a heparinized syringe. Deoxyribonucleic acid (DNA) was isolated according to the standard protocol (with chloroform and sodium perchlorate salting-out method). Polymerase chain reaction (PCR) amplification was performed for all coding exons including intron-exon boundaries of FGFR2 gene using primers published elsewhere. ${ }^{6}$ This was followed by direct resequencing through Sanger's method on an automated capillary sequencer (ABI-3130 genetic analyzer, Applied Biosystems, California, USA), as per the manufacturer's protocol.

\section{Case Report}

A 19 year-old boy suffering from Apert syndrome, with a novel clinical variant of bilateral tripod-shaped syndactyly, in addition to the typical features of craniosynostosis, dysmorphic face, and symmetrical syndactyly in both hands and feet, was recruited . The patient had milder craniofacial features together with hypertelorism, marked depression of the nasal bridge, long philtrum, and low-set ears. The patient's mental ability, cardiopulmonary function, dentition, palatal arch, and nasal cavity were normal (-Fig. 1).

\section{Results}

Direct resequencing of the all 18 full-length exons of the FGFR2 gene of this patient with Apert syndrome identified a mutation-FGFR2c.758C >G (rs77543610, FGFR2g.78299C >G, FGFR2p.P253R)-present in heterozygous form in the patient. This variant is previously reported in Apert syndrome and is a pathogenic, missense mutation present in the coding region of exon 7 of the gene that lies in the IgII-IgIII linker of the FGFR2 protein (-Fig. 2 ).

\section{Discussion}

Craniofacial dysmorphism and syndactyly of the hands and feet are the typical features of Apert syndrome. ${ }^{3}$ The present study had shown craniosynostosis, dysmorphic face with ocular hypertelorism, marked depression of the nasal bridge, long philtrum and low-set ear, and symmetrical syndactyly in both hands (tripod-shaped) and feet. The case in this article describes a novel clinical variant of Apert syndrome having a tripod-shaped symmetrical syndactyly, with milder craniofacial features.

Mutation in FGFR2 causes Apert syndrome. FGFR2 encodes a tyrosine kinase receptor which is activated by binding to FGF and plays a role in cell proliferation, angiogenesis, and bone differentiation mainly during embryonic development. ${ }^{9}$

In the present study, molecular genetic analysis of the FGFR2 in the present new variant of Apert syndrome showed a known missense mutation c.758C $>\mathrm{G}$ (p.Pro253Arg) in exon 7. It lies in a highly conserved region in the immunoglobulin-like extracellular subdomains of the FGFR2 gene. This is one of the two most common mutations in Apert syndrome. FGFR2c.758C $>\mathrm{G}$ mutation is reported to be associated with severe nonsyndromic syndactyly. ${ }^{9} \mathrm{FG}$ FR2c.758C >G (p.Pro253Arg) mutation was also reported in 3\% oncogenic cases of Apert syndrome, like squamous cell carcinoma (SCC) and endometrial carcinoma of the lung, head, and neck. ${ }^{4}$

Previous studies have shown that FGFR2c.755C $>$ G (p.Ser252Trp) mutation is strongly associated with a more severe craniofacial phenotype, limb defects, and cleft palate. ${ }^{4} F G$ FR2c.758C $>\mathrm{G}$ mutation in Apert syndrome was reported by Wilkie et al (1995). FGFR2c.755C >G (p.Ser252Trp) and FGFR2c.758C $>\mathrm{G}$ (p.Pro253Arg) are the two most common 
mutations in FGFR2 in Apert syndrome cases; they are reported in $\sim 85 \%$ cases of Apert syndrome..$^{4,9}$ These two adjacently positioned mutations lie in the linker between the second and third extracellular immunoglobulin (Ig) domains of FGFR2 protein. p.S252F. (exon7) and Alu-element insertion (within or near exon 9) are other common mutations identified in FGFR2 gene. ${ }^{4,5}$

\section{Conclusion}

In conclusion, the present study reports a novel clinical feature of Apert syndrome having a tripod-shaped symmetrical syndactyly, but with milder craniofacial features. This patient had FGFR2c.758C >G (p.Pro253Arg) mutation in exon 7, positioned in the IgII-IgIII linker of the FGFR2 protein which is one of the two most common mutations (p.Ser252Trp and p.Pro253Arg) in Apert syndrome.

\section{Conflict of Interest}

None declared.

\section{Acknowledgments}

The authors are grateful to the patient and family members for voluntary participation in the analysis. The authors are grateful to SERB, New Delhi, for a research grant to A. Ali, and to the Indian Council of Medical Research (ICMR) for providing Junior Research Fellowship (JRF) to the Ph.D. student CB Singh.

\section{References}

1 Wheaton SW. Two specimens of congenital cranial deformity in infants associated with fusion of the fingers and toes. Trans Pathol Soc London 1894;45:238

2 Apert ME. De I'acrocéphalosyndactylie. Bull MemSoc Med Hôp 1906;23:1310-1330

3 Brajadenta GS, Sari AIP, Nauphar D, Pratamawati TM, Thoreau V. Molecular analysis of exon 7 of the fibroblast growth factor receptor 2 (FGFR2) gene in an Indonesian patient with Apert syndrome: a case report. J Med Case Reports 2019;13(1):244

4 Kunwar F, Tewari S, Bakshi SR. Apert syndrome with S252W FGFR2 mutation and characterization using Phenomizer: an Indian case report. J Oral BiolCraniofac Res 2017;7(1):67-71

5 Kan SH, Elanko N, Johnson D, et al. Genomic screening of fibroblast growth-factor receptor 2 reveals a wide spectrum of mutations in patients with syndromiccraniosynostosis. Am J Hum Genet 2002;70(2):472-486

6 Panigrahi I. Craniosynostosis genetics: the mystery unfolds. Indian J Hum Genet 2011;17(2):48-53

7 Agrawal N, Frederick MJ, Pickering CR, et al. Exome sequencing of head and neck squamous cell carcinoma reveals inactivating mutations in NOTCH1. Science 2011;333(6046) :1154-1157

8 Liao RG, Jung J, Tchaicha J, et al. Inhibitor-sensitive FGFR2 and FGFR3 mutations in lung squamous cell carcinoma. Cancer Res 2013;73(16):5195-5205

9 Oldridge M, Lunt PW, Zackai EH, et al. Genotype-phenotype correlation for nucleotide substitutions in the IgII-IgIII linker of FGFR2. Hum Mol Genet 1997;6(1):137-143

10 Oldridge M, Zackai EH, McDonald-McGinn DM, et al. De novo Alu-element insertions in FGFR2 identify a distinct pathological basis for Apert syndrome. Am J Hum Genet 1999;64(2):446-461 